\title{
The Role of RNA Interference in Functional Cure Strategies for Chronic Hepatitis B
}

\author{
Jeremy S Nayagam ${ }^{1,2}$ (D) Zillah C Cargill $^{1}$ (D) $\cdot$ Kosh Agarwal $^{1}$ (D)
}

Accepted: 16 October 2020 / Published online: 28 October 2020

(C) The Author(s) 2020

\begin{abstract}
Purpose of Review Current treatments for chronic hepatitis B (CHB) are associated with low rates of cure. Functional cure has been accepted as a viable treatment end point in CHB. There have been substantial advances in the field of RNA interference (RNAi) therapeutics across a wide range of specialties, and the clinical pipeline now encompasses CHB. This review will highlight some of the challenges in therapeutic development, the data for RNAi in CHB, and future directions for the field.

Recent Findings Early phase clinical trials have reported good safety data for RNAi therapies in CHB and demonstrated significant reductions in quantitative HBsAg levels (qHBsAg). Animal models however suggest that in HBeAg-negative individuals, HBsAg may be derived from hepatitis B DNA integrated into the host genome, which cannot be targeted by current RNAi therapies, and may prove to be a limitation. Preliminary data is being presented from combination therapy, which may result in more robust reductions in qHBsAg; however, trials are in the early stages of recruitment.

Summary Despite promising data that RNAi may be an effective therapeutic strategy in CHB, it is unlikely to be in the form of monotherapy. The goal for the community will be to find the right combination of RNAi therapy with other antiviral or immunomodulatory agents, to achieve functional cure with a cessation of therapy. Early phase clinical trials are continuing to recruit, and data from combination studies will provide a "pivot point" in determining whether RNAi therapies can provide a backbone to finite duration and curative $\mathrm{CHB}$ regimens.
\end{abstract}

Keywords Hepatitis B $\cdot$ RNA interference $\cdot$ Functional cure $\cdot$ siRNA

\section{Introduction}

Chronic hepatitis B infection (CHB) is a global epidemic estimated to affect over 257 million people and is one of the leading causes of deaths from liver-related deaths and hepatocellular carcinoma (HCC) [1]. The World Health Assembly has provided goals and metrics to eliminate viral hepatitis as a public health problem. Despite encouraging steps, with increasing uptake of the hepatitis B virus (HBV) vaccine and prevention of new cases of $\mathrm{CHB}$, these focus only on the

This article is part of the Topical Collection on Hepatitis $B$

Jeremy S Nayagam

j.nayagam@nhs.net

1 Institute of Liver Studies, King's College Hospital, London SE5 9RS, UK

2 Department of Inflammation Biology, King's College London, London, UK prevention aspects and do not impact on the liver-related complications in those with existing CHB. Not only are the majority of individuals unaware that they are living with $\mathrm{CHB}$, an even smaller minority are receiving treatment [1].

The annual rate of hepatitis B surface antigen (HBsAg) seroclearance is approximately $1 \%$, with no difference between untreated individuals and those who have received antiviral therapy [2]. Antiviral therapy is not required in all patients with $\mathrm{CHB}$, and longitudinal monitoring is important in risk stratification. The goal of therapy is to reduce the risk of progression to cirrhosis, end-stage liver disease, and HCC in selected individuals [3]. The two currently available treatment options for CHB are nucleot(s)ide analogues (NA) and pegylated interferon (PEG-IFN). Second-generation NA are widely utilized; they are highly effective in controlling viral replication and are associated with reduced adverse outcomes; however, they do not interfere with viral antigen production or secretion as they work in the late stages of replication in the $\mathrm{HBV}$ cycle. Related to this mechanism of action, cure of CHB with NA therapy is rare. PEG-IFN can induce long-term 
immunological control but due to the extensive side effect profile, patient selection is vital, and it can only be used in those with mild or moderate liver disease due to the risk of a hepatitis flare and resultant decompensation [3]. In addition, there is limited evidence of an additional benefit from combination of NA- and IFN-based therapies, and it appears to be a regimen that is poorly tolerated $[3,4]$.

\section{What Is a Functional Cure?}

A better understanding of the HBV life cycle has paved the way for a rapid expansion of the therapeutic targets and novel antivirals in clinical trials. A key juncture in the roadmap for hepatitis B antiviral therapies was the workshop convened by the American Association for the Study of Liver Diseases and the European Association for the Study of the Liver in 2016, where leading stakeholders were convened to develop a consensus on treatment endpoints. Three definitions were proposed: complete sterilizing cure (undetectable HBsAg in serum and eradication of intrahepatic cccDNA and integrated HBV DNA), functional cure (sustained undetectable HBsAg and HBV DNA in serum after a finite course of treatment, with or without seroconversion to anti-HBs), and partial cure (detectable HBsAg but persistently undetectable HBV DNA in serum after a finite course of treatment). Of the three definitions, the majority supported the adoption of functional cure as the definition of cure, in part as achieving complete sterilizing cure is not thought to be feasible [5॰].

The rates of functional cure with currently available therapies are relatively low, but are associated with improved clinical liver-related outcomes. From a large study of patients with CHB treated with NA, overall rate of HBsAg seroclearance was $2 \%$ [6]. The 8-year cumulative incidence rate of HCC in those with functional cure was $0.6 \%$, and this was a lower risk compared to those who only achieve complete viral suppression with NA therapy. PEG-IFN monotherapy for 48 weeks achieved HBsAg seroclearance in 3\% of individuals, which was superior to NA monotherapy with lamivudine [7]. From pooled data of patients who received NA, PEG-IFN, or a combination of both, once HBsAg seroclearance was achieved, it was durable in $82 \%$ of cases [8]. Individuals who achieved HBsAg seroclearance with IFN-based therapies demonstrated a peak recurrence at 52 weeks, which was seen in only $6.3 \%$ of individuals [9].

It is important to identify individuals who will derive a benefit from achieving a functional cure and possible limitations. In patients with $\mathrm{CHB}$ and compensated cirrhosis, HBsAg seroclearance, either with IFN therapy or spontaneous, was associated with a lower incidence of HCC and improved survival compared to those do did not achieve HBsAg seroclearance [10]. Large-scale longitudinal studies evaluating outcomes in patients who have achieved HBsAg seroclearance have identified those who achieved HBsAg at an older age (over 50 years), who are males, and those with cirrhosis are at a higher risk of developing HCC and may benefit from ongoing surveillance [11, 12]. In NA-induced functional cure, there was no difference in hepatic events or mortality, between those who achieved functional cure and those with complete viral suppression [6].

\section{What Is RNA Interference?}

RNA interference (RNAi) is a highly specific and efficient method of post-transcriptional gene silencing. It is a biologically conserved mechanism found across a large number of organisms $[13,14]$. Manipulation of its pathways with therapeutic agents to target sequence-specific mRNAs of diseaserelated genes has huge potential for the treatment of multiple conditions [15•].

Initiation of this process is by double-stranded RNAs (dsRNAs) in the cytoplasm of the cell, which can originate from both endogenous and exogenous pathways (Fig. 1) [13, 16]. These long dsRNAs are cleaved by the ribonuclease protein Dicer into short fragments (20-30 nt), termed small interfering RNAs (siRNAs), and comprise of a "guide" strand (antisense) and a "passenger" strand (sense). The siRNA is separated into single strands, and the guide strand binds to the Argonaute 2 protein. The passenger strand is degraded, and subsequently, the RNA-induced silencing complex (RISC) assembles. The guide strand has a perfect, or near perfect, complementary sequence to the target RNA. Therefore, there is homology-dependent recognition of the target RNA when it enters the RISC. The gene silencing response then ensues. Depending on the structure of the siRNA and biological context, different mechanisms of silencing can be induced. Methods include cleavage of the target complementary mRNA within the RISC or sequestration of the mRNA, preventing binding to translational ribosomal RNAs. The active RISC can go on to further target multiple mRNA molecules for degradation.

\section{Why Is RNAi Attractive in CHB?}

Drug candidates for RNAi therapeutics are being developed for multiple organs, but the majority target the liver where the greatest advances in the field have been made [15•]. Patisiran (Alnylam Pharmaceuticals) is an RNAi agent, which inhibits hepatic production of transthyretin and improves clinical manifestations in patients with hereditary transthyretin, and was the first RNAi therapeutic approved by the US Food and Drug Administration (FDA) $[17 \cdot \bullet]$. A recent phase 3 trial evaluating givosiran (Alnylam Pharmaceuticals), an RNAi agent that targets hepatic ALA 
Fig. 1 RNAi silencing mechanism in mammals. 1 . (a) Exogenous dsRNA or shRNA enters the cytoplasm via endocytosis. (b) Endogenous premiRNA is exported out of the nucleus. 2. These interact with RNA interference enzymes Dicer and TAR RNA-binding protein (TRBP), resulting in their cleavage. Non-Dicer-mediated cleavage pathways are also possible. 3. Arogonaute proteins bind to form the RNA-induced silencing complex (RISC) Loading Complex. siRNA is unwound, and the guide strand is selected and loaded into Ago. 4. The mature RISC compromises of Ago, GW182, and the loaded guide RNA strand. Dicer and TRBP dissociate after its formation. 5. RISC is able to regulate gene expression by inhibiting translation of the target mRNA. This can occur via a number of mechanisms, including mRNA sequestration in the cytoplasm or mRNA degradation. 6. Due to this, the gene cannot be translated, and therefore, expression of the encoded protein is prevented.
1. Exogenous RNAi

Endogenous RNAi

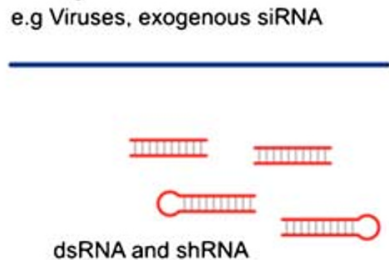

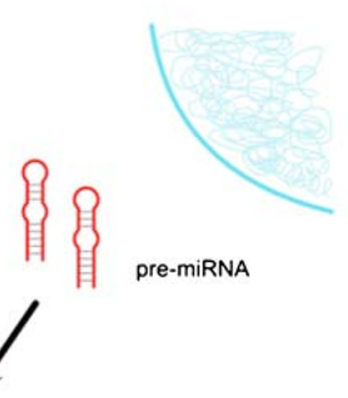

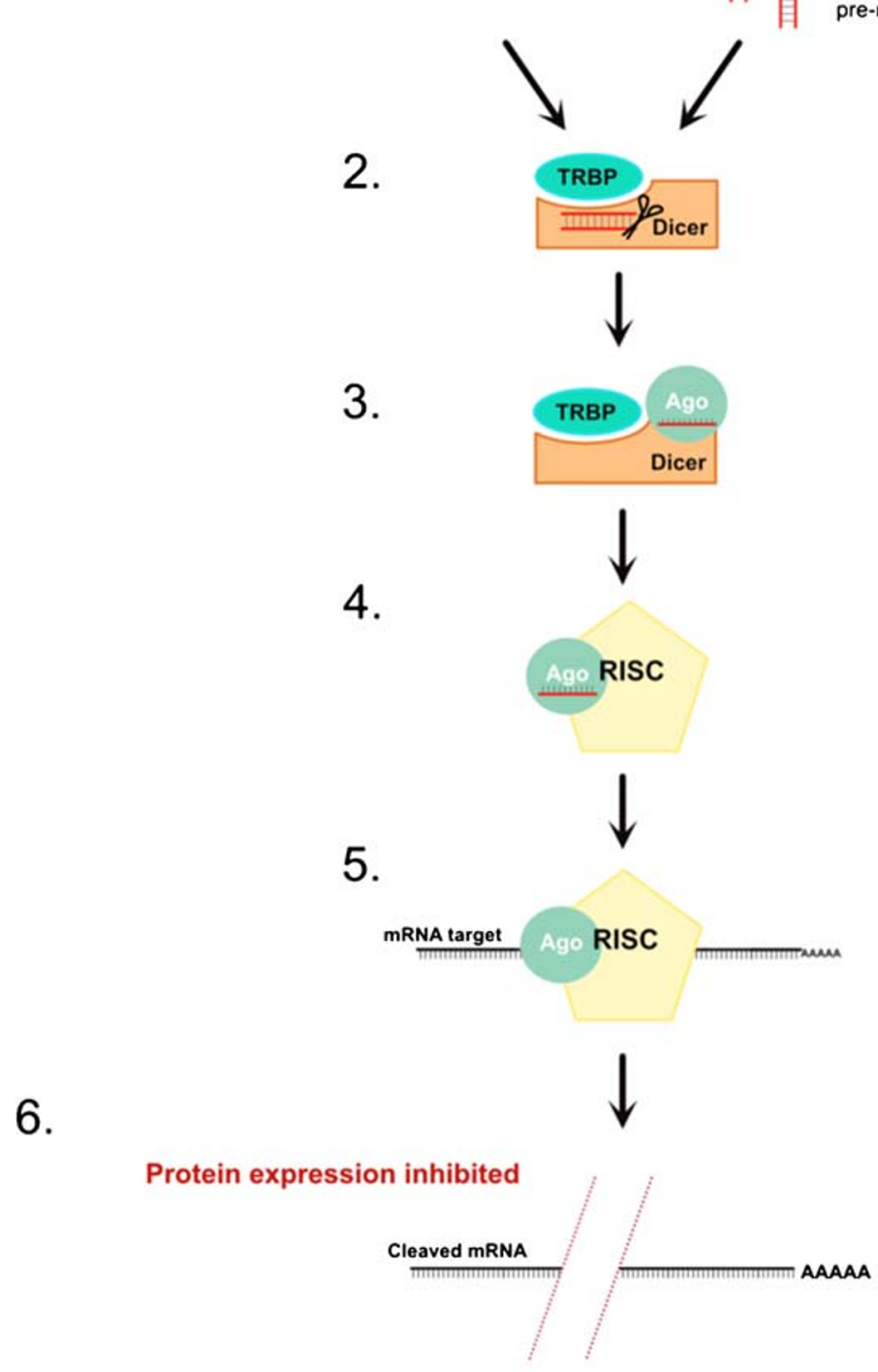

synthase 1 , in patients with acute intermittent porphyria has reported that it has demonstrated excellent safety and efficacy data and has also been approved by the FDA [18••]. These trials highlighting how RNAi therapeutics target the liver are now a realistic therapeutic strategy, and innovations from the field can be adapted for use in other liver conditions such as CHB. The complexity of HBV life cycle, its integration into the host genome, and its effect on the host immune system all contribute to the challenges in finding a functional cure for CHB. The use of an RNAi-based approach has multiple potential benefits and may be able to harness natural mechanisms of RNAi to target a variety of sites integral to CHB.

The molecular biology of the HBV means that it is amenable to therapeutic RNAi and can be used to target the production of viral proteins. The HBV virion is $3.2 \mathrm{~kb}$ pairs in length and has a compact structure. Once within the nucleus of the hepatocyte, host DNA repair mechanisms are involved in the conversion into a covalently closed circular DNA (cccDNA), which is an important factor in HBV transcription and replication. cccDNA is used as a template by host RNA 
polymerase II on four open reading frames to transcribe viral RNA which encode the proteins: precore/core, polymerase, surface, and X [19]. All four transcripts are encoded in overlapping reading frames with a common $3^{\prime}$ end. This makes RNAi an attractive therapeutic option, as a single RNAi can potentially result in the degradation of all the viral transcripts and thus all the viral proteins. Although cccDNA is not directly acted upon by RNAi, the cccDNA reservoir in the nucleus may be diminished as a consequence of viral replication inhibition [19].

$\mathrm{CHB}$ has a profound effect on $\mathrm{T}$ cell function, and although $\mathrm{T}$ cells are activated, they are functionally exhausted and have a limited ability to proliferate $[20,21]$. The prolonged exposure of $\mathrm{T}$ cells to a high antigen load is believed to maintain this $\mathrm{T}$ cell dysfunction and contribute to chronicity. The ability of RNAi to directly target RNA involved in the production of viral antigens is an appealing prospect in RNAi for CHB. NA decreases polymerase-reverse transcriptase and decreases viral titers, but is less effective at reducing viral antigens in most patients. RNAi may allow a faster and more robust reduction in viral antigens, with associated benefits at an immune level, reconstituting specific antigen directly targeting host immune responses.

\section{Challenges in the Development of RNAi for CHB}

Despite the theoretical role for utilizing RNAi in CHB, there are a number of key factors in the design and development of RNAi drugs for CHB. A selection of the critical challenges related to ensuring delivery of RNAi to the target cells while avoiding toxicity is outlined below.

RNAi requires the systemic administration of therapeutic molecules with an effective delivery system to reach the target cells. This can be achieved using a viral or non-viral delivery system [22]. Advances have been made with both techniques; however, concerns exist regarding immunogenicity and risk of mutations when using viral vectors. The use of a stable hydrophilic non-interactive agent, such as polyethylene glycol (PEG), was an important advance in the field and allows systemic delivery of RNAi with minimal non-specific interaction en route to the liver [22]. The challenges associated with the pharmacokinetic properties of drugs have improved with lipid and polymer nanoparticles technology; however, it remains an important consideration in achieving a therapeutic window in the required tissue with systemic delivery and a feasible dosing frequency $\left[15^{\bullet}\right]$.

Upon reaching the liver, the molecules must exit the intravascular space to reach the hepatocytes. As the cells are fenestrated, particles $<100 \mathrm{~nm}$ in diameter can exit the hepatic vasculature and interact with liver parenchymal cells [23]. Liposomes must avoid uptake by, and activation of, Kuppfer cells, which are specialized macrophages that line the sinusoids [23]. This can be achieved by labeling liposomes with ligands to target specific receptors on hepatocytes. Asialoglycoprotein receptor (ASGP-R) is an abundant receptor on the hepatocytes that recognizes proteins with an exposed terminal galactose or $N$-acetylgalactosamine residue and internalizes them [24], and can be exploited for drug delivery [23]. The use of $\mathrm{N}$-acetylgalactosamine (GalNAc) as a conjugate is being extensively investigated as it avidly binds to ASGP-R and may be the solution to ensuring targeted delivery to the hepatocytes $\left[25^{\circ}\right]$.

The molecules need to enter the hepatocytes to elicit RNAi. Once attached to the surface of the hepatocyte, the complexes enter via receptor-mediated endocytosis [15•]. ASGP-R binds to ligands at a neutral $\mathrm{pH}$ of the hepatocyte surface and then releases them in the acidic environment of the endosome which allows the active molecules to escape endocytosis $[26 \bullet \bullet$.

In parallel to ensuring delivery of the molecule to the target tissue, it is vital to avoid non-specific toxicity from the RNAi molecule. The four main pathways for toxicity are immunogenic reactions to dsRNA, toxicity of excipients chemicals, unintended RNAi activity in non-target genes, and on-target RNAi activity in non-target tissue [15•]. Protein kinase R (PKR), Toll-like receptor 3 (TLR3), and TLR7-mediated sensing of dsRNAs are associated with the immunogenic reaction, although $2^{\prime}$ sugar modifications have abrogated this cytotoxicity without impacting on gene silencing function [27]. As outlined, a number of excipient chemicals are required for effective drug delivery; however, either directly from the components themselves or from their metabolic breakdown, dose-limiting toxicities are seen [15•]. A particular challenge is in pinpointing the precise toxic component, and therefore, it has been suggested that future therapies are limited to a small number of excipients with confirmed low toxicity [15•].

RNAi is based on the near perfect match of the short sequence on the guide strand and the target RNA; however, there can be inadvertent matches with the RNAi guide strand and non-target RNA leading to unintended RNAi activity [28]. By screening potential target sites against the human genome, those with significant overlap can be excluded as candidates; however, this does not negate the need for close testing in primate models [15 ]. Despite these measures, a degree of overlap is unavoidable, so doses are minimized while the benefit of novel base pairs is explored [15•]. Systemic administration of the therapeutic molecule can result in its accumulation in non-target tissue, with associated toxicity in healthy tissue [15•]. A combination of careful selection of highly specific genes for the disease and individualized delivery routes can minimize this risk. Despite these measures, unintended RNAi activity cannot be avoided, and 
the recent progress with antisense oligonucleotide development, which can reverse drug activity in vivo, is an exciting prospect for drug safety [29].

\section{RNAi Treatments in CHB}

A number of pharmaceutical companies now include RNAi therapeutics in their clinical pipelines, which employ a variety of delivery platforms and adopt expertise from RNAi for other conditions. RNAi for CHB poses challenges related to the viral and clinical heterogeneity of patients, such as $\mathrm{HBeAg}$ status and prior treatments, which are addressed in different cohorts in the clinical trials, and subgroup analysis will provide important information. With the field now expanding to combination and potentially sequential therapies, defining the optimal timing of commencing RNAi drugs will be a key factor in determining its efficacy and role in the armamentarium.

ARC-520 (Arrowhead Pharmaceuticals) consists of 2 siRNAs conjugated to cholesterol and employs a polymerbased system (Dynamic PolyConjugates, DPC ${ }^{\mathrm{TM}}$ ) which is only activated in the acidic environment of the endosome, and allows endosomal escape and cytoplasmic delivery. It was the first RNAi therapeutic to publish data for CHB, with the phase 1 data utilizing a single dose of the intravenous preparation associated with a favorable tolerability profile [26••]. The phase 2 trial of $1-2$ doses of ARC-520 reported a comparable incidence of adverse events between treatment and placebo groups (67\%), and similar to the phase 1 trial, there was a beneficial role for oral antihistamine pre-treatment [30••]. The reduction in qHBsAg was seen across all groups, with a stronger reduction in treatment naïve $\mathrm{HBeAg}$-positive patients ( $1.4 \log _{10} \mathrm{IU} / \mathrm{mL}$ from baseline) and less so in $\mathrm{HBeAg}$ negative and treatment experienced with NA $\left(0.3 \log _{10} \mathrm{IU} /\right.$ $\mathrm{mL})[30 \bullet \cdot]$. There was a relapse in qHBsAg levels at 68 weeks post dose. In the phase 2 trials of ARC-520 in NA experienced with $\mathrm{CHB}$ who received four monthly doses alongside their standard NA, there was a significant reduction in qHBsAg 30 days after the last dose in both $\mathrm{HBeAg}$-positive and $\mathrm{HBeAg-negative} \mathrm{patients} \mathrm{compared} \mathrm{to} \mathrm{baseline} \mathrm{in} \mathrm{the}$ high-dose group [31・0]. Although this persisted for approximately 85 days after the last dose, the mean reduction in qHBsAg was only modest when compared to placebo, at 0.38 and $0.54 \log _{10} \mathrm{IU} / \mathrm{mL}$, respectively, for $\mathrm{HBeAg}$ positive and $\mathrm{HBeAg}$-negative cohorts.

ARB-1467 (Arbutus Biopharma) is an intravenous preparation which includes 3 siRNAs and utilizes a proprietary lipid nanoparticle platform [32]. The data from the 8-week multidose phase 2a trial in HBeAg-negative CHB patients who were suppressed with NA has been reported, but only in abstract form [33]. The therapy was generally well tolerated, with mostly mild or transient adverse events reported. All subjects achieved a reduction in qHBsAg from baseline, with a greater reduction seen in those who received more frequent dosing and at higher doses (mean 0.6 to $1.2 \log _{10} \mathrm{IU} / \mathrm{mL}$ in different cohorts); however, due to the necessity of intravenous delivery, this is not being taken forward clinically.

There are a number of ongoing trials in CHB involving RNAi, which have now progressed to subcutaneous preparations and are building on the innovations in the field and fine tuning the delivery platforms to optimize safety and efficacy (Table 1). LUNAR-HBV (Arcturus Therapeutics, Janssen Pharmaceuticals) has shown potent activity in pre-clinical studies using unlocked nucleomonomer agent oligomers (UNA) with their LUNAR ${ }^{\mathrm{TM}}$ delivery technology [34] and is yet to commence clinical trials. DCR-HBVS (Dicerna Pharmaceuticals, Roche) is in phase 1 trials (NCT03772249) and utilizes their GalXC' ${ }^{\text {TM }}$ technology and tetraloop configuration which may allow stronger potency and a longer duration of action. AB-729 (Arbutus Biopharma) uses a covalently conjugated GalNAc delivery technology and is in phase $1 \mathrm{a} / 1 \mathrm{~b}$ trial from which they have announced preliminary safety data from the single-dose cohort and are planning to proceed with multiple dosing (company website). VIR-2218 (Alnylam Pharmaceuticals, Vir Biotechnology) uses an Enhanced Stabilization Chemistry-Plus (ESC+)-GalNAc delivery platform and is in phase 1 and 2 trials (NCT03672188), which are due to complete in March 2021. IONIS-HBVRx (Ionis Pharmaceuticals, GlaxoSmithKline) uses antisense oligonucleotide technology, which identified no safety concerns from their phase 1 data [35]. They are currently in phase 2 trials in CHB (NCT02981602), and it is a platform which incidentally has recently reported encouraging phase 2 data in nonalcoholic liver disease [36••].

ARO-HBV, now JNJ-3989 (Arrowhead Pharmaceuticals, Janssen Pharmaceuticals), has evolved from the first-generation ARC-520 which employed the DPCTM delivery and instead uses direct conjugation for hepatocyte delivery with their TRIM $^{\mathrm{TM}}$ platform. It is a subcutaneous formulation containing two siRNAs, with data from 40 patients who received 3 doses in combination with NA reporting it was well tolerated. It achieved $>1 \log _{10} \mathrm{IU} / \mathrm{mL}$ reduction in $\mathrm{qHBsAg}$ at 56 days post last dose in $97 \%$ of those who received a dose of $100 \mathrm{mg}$ or higher [37]. It is now in phase $2 \mathrm{~b}$ placebo-controlled clinical trials in different combination regimens $(40 \mathrm{mg}$, $100 \mathrm{mg}$, and $200 \mathrm{mg}$ doses with NA and with or without capsid assembly molecules (CAM) for 48 weeks (NCT03982186); and placebo controlled with $200 \mathrm{mg}$ dose in combination with NA and CAM compared to NA monotherapy for 48 weeks in NA suppressed (NCT04129554)). The experience from the first 12 patients using the combination regimen of JNJ-3989 (200 mg), CAM and NA, with follow-up 2 months post last dose of RNAi, did not identify a safety concern [38]. 
Table 1 The pipeline of clinical trials of RNAi for chronic hepatitis B

\begin{tabular}{|c|c|c|}
\hline RNAi therapeutic & Delivery system and technology & Clinical trial status \\
\hline $\begin{array}{l}\text { LUNAR-HBV (Arcturus Therapeutics, } \\
\text { Janssen Pharmaceuticals) }\end{array}$ & $\begin{array}{l}\text { Unlocked nucleomonomer agent oligomer, } \\
\text { LUNAR }{ }^{\mathrm{TM}} \text { delivery technology }\end{array}$ & Yet to commence \\
\hline $\begin{array}{l}\text { DCR-HBVS (Dicerna Pharmaceuticals, } \\
\text { Roche) }\end{array}$ & $\begin{array}{l}\text { GalXC }{ }^{\mathrm{TM}} \text { technology and tetraloop } \\
\text { configuration }\end{array}$ & Phase 1 -active (NCT03772249) \\
\hline AB-729 (Arbutus Biopharma) & Covalently conjugated GalNAc delivery & $\begin{array}{l}\text { Phase } 1 \mathrm{a} / 1 \mathrm{~b} \text { - preliminary safety data on company website, } \\
\text { active }\end{array}$ \\
\hline $\begin{array}{l}\text { VIR-2218 (Alnylam Pharmaceuticals, } \\
\text { Vir Biotechnology) }\end{array}$ & $\begin{array}{l}\text { Enhanced Stabilization Chemistry-Plus } \\
\text { (ESC+)-GalNAc delivery platform }\end{array}$ & Phase 1 and 2 -active (NCT03672188) \\
\hline $\begin{array}{l}\text { IONIS-HBVRx (Ionis Pharmaceuticals, } \\
\text { GlaxoSmithKline) }\end{array}$ & Antisense oligonucleotide technology & $\begin{array}{l}\text { Phase } 1 \text { - safety data [35] } \\
\text { Phase } 2 \text { - active (NCT02981602) }\end{array}$ \\
\hline $\begin{array}{l}\text { JNJ-3989 (Arrowhead Pharmaceuticals, } \\
\text { Janssen Pharmaceuticals) }\end{array}$ & Direct conjugation, TRIM ${ }^{\mathrm{TM}}$ platform & $\begin{array}{l}\text { Phase } 2 \mathrm{~b} \text { - safety data }[37,38] \text {, active in combination with } \\
\text { CAM (NCT03982186, NCT04129554) }\end{array}$ \\
\hline
\end{tabular}

Reductions in qHBsAg of $>1 \log _{10}$ IU $/ \mathrm{mL}$ (mean $1.7 \log _{10} \mathrm{IU} / \mathrm{mL}$ ) were seen in all patients after 3 doses of JNJ-3989, which were similar in HBeAg-positive and HBeAg-negative cohorts, and the effect persisted for over 2 months after the last dose [38].

Important lessons need to be learnt from the early trials in order to improve future therapeutics and trial designs. The differential response seen in certain subgroups from the ARC-520 trial prompted further investigation in chimpanzees with chronic hepatitis B [30••]. There is a suggestion that HBsAg is not only expressed from cccDNA but also from transcripts arising from HBV DNA integrated into the host genome, which was the dominant source in HBeAg-negative chimpanzees [30••]. The therapeutic siRNA could not target the mRNA derived from integrated HBV DNA. This suggests that there may be changes needed in trial design and end point expectations and that RNAi therapeutics need to be able to target viral transcripts regardless of origin [30••, 31].

\section{Conclusions and Future Perspectives}

Chronic hepatitis B remains a global health issue. Despite the availability of therapies effective in the reduction of clinical liver-related complications in CHB, there are many limitations, including the low rates of what can be defined as functional cure. The molecular biology of HBV makes it an attractive target for more upstream therapies. RNAi harnesses natural pathways to induce gene silencing and has already been licensed in rare genetic liver disease with an excellent safety profile. In CHB, the use of RNAi could potentially reduce viral antigen production more than NA therapies, with beneficial effect on immune exhaustion seen in $\mathrm{CHB}$. There are many challenges in developing RNAi for CHB therapies, and there are currently no licensed therapies, although the safety profile in early phase studies has been acceptable. Despite promising data in early phase clinical trials, significant sustained reductions in qHBsAg have not been demonstrated, and no medium- or long-term data off treatment has been reported. Both of these are essential to determine therapeutic efficacy and are eagerly awaited from later phase trials. The trials to date have reported $\mathrm{qHBsAg}$ as a surrogate marker for ontreatment efficacy, which is largely drawn from predictors of response to IFN [3]. With the different therapeutic mechanism of RNAi, however, it may be that biomarkers more sensitive for cccDNA transcription, such as $\mathrm{HBcrAg}$ and HBV RNA, will be more accurate in predicting response to RNAi and may need to be incorporated into trial design [39]. A recent abstract of a small subgroup who received a short course of RNAi (JNJ-3989) in combination with NA suggests that reductions in these biomarkers may correlate with a sustained qHBsAg response [40].

To deliver the benchmark of functional cure in $\mathrm{CHB}$, it is likely that different mode of action therapies for a finite duration will be required and perhaps tailored for viral and host characteristics, including $\mathrm{HBeAg}$ status. While the data is rapidly evolving, and heterogeneity in responses has been reported, it is possible that RNAi could be a cornerstone of therapy with NA in CHB. However, there are multiple RNAi in early development and it is too early to predict future regimens. Combination therapy studies with RNAi are advancing rapidly, with the most advanced being JNJ-3989 with CAM and NA in phase 2 b studies. The agents in this trial all target the viral life cycle, and currently, antiviral agents are dominating the trial landscape. A strategy of combination, induction, or sequential therapy with RNAi, with or without an immune target (such as a checkpoint inhibitor) with an additional antiviral or entry inhibitor, would provide a fascinating insight into the potential future landscape. It is paramount that while striving for functional cure with combinations of novel therapies, a favorable side effect profile is maintained and that treatments are applicable to a global CHB population. 
Authors' Contributions Not applicable.

Funding No funding was received to support this publication.

Data Availability Not applicable.

\section{Compliance with Ethical Standards}

Conflict of Interest JSN no disclosures; ZCC no disclosures; KA (i) Consultancy/Speaker bureau: Assembly, Aligos, Arbutus, Gilead, Immunocore, Janssen, Roche, Sobi, Shinoigi, and Vir (ii) Grant support: Abbott, Gilead, MSD.

Ethics Approval Not applicable.

Consent to Participate Not applicable.

Consent for Publication Not applicable.

Code Availability Not applicable.

Open Access This article is licensed under a Creative Commons Attribution 4.0 International License, which permits use, sharing, adaptation, distribution and reproduction in any medium or format, as long as you give appropriate credit to the original author(s) and the source, provide a link to the Creative Commons licence, and indicate if changes were made. The images or other third party material in this article are included in the article's Creative Commons licence, unless indicated otherwise in a credit line to the material. If material is not included in the article's Creative Commons licence and your intended use is not permitted by statutory regulation or exceeds the permitted use, you will need to obtain permission directly from the copyright holder. To view a copy of this licence, visit http://creativecommons.org/licenses/by/4.0/.

\section{References}

Papers of particular interest, published recently, have been highlighted as:

- Of importance

•- Of major importance

1. World Health Organization. Global Hepatitis Report 2017.

2. Liaw YF, Sheen IS, Chen TJ, Chu CM, Pao CC. Incidence, determinants and significance of delayed clearance of serum HBsAg in chronic hepatitis B virus infection: a prospective study. Hepatology. 1991;13(4):627-31.

3. European Association For The Study Of The Liver. EASL 2017 clinical practice guidelines on the management of hepatitis B virus infection. J Hepatol. 2017;67(2):370-98.

4. Bourlière M, Rabiega P, Ganne-Carrie N, et al. Effect on HBs antigen clearance of addition of pegylated interferon alfa-2a to nucleos $(t)$ ide analogue therapy versus nucleos $(t)$ ide analogue therapy alone in patients with $\mathrm{HBe}$ antigen-negative chronic hepatitis B and sustained undetectable plasma hepatitis B virus DNA: a randomised, controlled, open-label trial. Lancet Gastroenterol. 2017;2(3):177-88.

5. Lok AS, Zoulim F, Dusheiko G, Ghany MG. Hepatitis B cure: from discovery to regulatory approval. J Hepatol. 2017;67(4):847-61
This paper outlines the agreed treatment endpoints for clinical trials in chronic hepatitis $B$.

6. Yip TCF, Wong GLH, Chan HLY, Tse YK, Lam KLY, Lui GCY, et al. HBsAg seroclearance further reduces hepatocellular carcinoma risk after complete viral suppression with nucleos (t) ide analogues. J Hepatol. 2019;70(3):361-70.

7. Lau GK, Piratvisuth T, Luo KX, Marcellin P, Thongsawat S, Cooksley G, et al. Peginterferon Alfa-2a, lamivudine, and the combination for HBeAg-positive chronic hepatitis B. N Engl J Med. 2005;352(26):2682-95.

8. Lok AS, Zoulim F, Dusheiko G, Chan HLY, Buti M, Ghany MG, et al. Durability of hepatitis B surface antigen loss with nucleotide analogue and peginterferon therapy in patients with chronic hepatitis B. Hepatol Comm. 2020;4(1):8-20.

9. Wu Y, Liu Y, Lu J, Cao Z, Jin Y, Ma L, et al. Durability of interferon-induced hepatitis B surface antigen seroclearance. Clin Gastroenterol Hepatol. 2020;18(2):514-6.

10. Fattovich G, Giustina G, Sanchez-Tapias J, Quero C, Mas A, Olivotto PG, et al. Delayed clearance of serum HBsAg in compensated cirrhosis B: relation to interferon alpha therapy and disease prognosis. Am J Gastroenterol. 1998;93(6):896-900.

11. Yuen MF, Wong DKH, Fung J, et al. HBsAg Seroclearance in chronic hepatitis B in Asian patients: replicative level and risk of hepatocellular carcinoma. Gastroenterology. 2008;135(4):1192-9.

12. Kim GA, Lim YS, An J, Lee D, Shim JH, Kim KM, et al. HBsAg seroclearance after nucleoside analogue therapy in patients with chronic hepatitis B: clinical outcomes and durability. Gut. 2014;63(8):1325-32.

13. Agrawal N, Dasaradhi PVN, Mohmmed A, Malhotra P, Bhatnagar RK, Mukherjee SK. RNA interference: biology, mechanism, and applications. Microbiol Mol Biol Rev. 2003;67(4):657-85.

14. Mello CC, Conte D. Revealing the world of RNA interference. Nature. 2004:431:338-42.

15. Setten RL, Rossi JJ, Han SP. The current state and future directions of RNAi-based therapeutics. Nat Rev Drug Discov. 2019;18(6): 421-46 This excellent review article outlines potential toxicity with RNAi and the horizon for therapeutic RNAi.

16. Hannon GJ. RNA interference. Nature. 2002;418(6894):244-51.

17.• Adams D, Gonzalez-Duarte A, O'Riordan WD, et al. Patisiran, an RNAi therapeutic, for hereditary transthyretin amyloidosis. N Engl J Med. 2018;379(1):11-21 This is a landmark study of Patisiran, the first RNAi therapeutic approved by the FDA, for the use in hereditary transthyretin amyloidosis.

18.• Balwani M, Sardh E, Ventura P, et al. Phase 3 trial of RNAi therapeutic Givosiran for acute intermittent porphyria. N Engl J Med. 2020;382:2289-301 This recent study has demonstrated safety and efficacy of Givosiran an RNAi for acute intermittent porphyria, which has also been approved by the FDA.

19. Gish RG, Yuen MF, Chan HLY, Given BD, Lai CL, Locarnini SA, et al. Synthetic RNAi triggers and their use in chronic hepatitis B therapies with curative intent. Antivir Res. 2015;121:97-108.

20. Boni C, Fisicaro P, Valdatta C, Amadei B, di Vincenzo P, Giuberti $\mathrm{T}$, et al. Characterization of hepatitis B virus (HBV)-specific T-cell dysfunction in chronic HBV infection. J Virol. 2007;81(8):421525 .

21. Bertoletti A, Ferrari C. Innate and adaptive immune responses in chronic hepatitis B virus infections: towards restoration of immune control of viral infection. Gut. 2012;61(12):1754-64.

22. Castanotto D, Rossi JJ. The promises and pitfalls of RNAinterference-based therapeutics. Nature. 2009;457(7228):426-33.

23. Wu J, Nantz MH, Zern MA. Targeting hepatocytes for drug and gene delivery: emerging novel approaches and applications. Front Biosci. 2002;7(3):717-25.

24. Stockert RJ. The asialoglycoprotein receptor: relationships between structure, function, and expression. Physiol Rev. 1995;75(3):591609. 
25. Springer AD, Dowdy SF. GalNAc-siRNA conjugates: leading the way for delivery of RNAi therapeutics. Nucleic Acid Ther. 2018;28(3):109-18 This article outlines the barriers to delivering RNAi to hepatocytes, and the successful development of the GalNAc conjugate to overcome this.

26.• Schluep T, Lickliter J, Hamilton J, et al. Safety, tolerability, and pharmacokinetics of ARC-520 injection, an RNA interferencebased therapeutic for the treatment of chronic hepatitis B virus infection, in healthy volunteers. Clin Pharm Drug Dev. 2017;6(4): 350-62 This is the first phase 1 study of RNAi therapeutics in chronic hepatitis $B$ and reports promising safety data.

27. Robbins M, Judge A, Liang L, McClintock K, Yaworski E, MacLachlan I. 2'-O-methyl-modified RNAs act as TLR7 antagonists. Mol Ther. 2007;15(9):1663-9.

28. Janas MM, Schlegel MK, Harbison CE, et al. Selection of GalNAcconjugated siRNAs with limited off-target-driven rat hepatotoxicity. Nat Commun. 2018;9(1):1-10.

29. Zlatev I, Castoreno A, Brown CR, Qin J, Waldron S, Schlegel MK, et al. Reversal of siRNA-mediated gene silencing in vivo. Nat Biotechnol. 2018;36(6):509-11.

30.• Wooddell CI, Yuen MF, HLY C, et al. RNAi-based treatment of chronically infected patients and chimpanzees reveals that integrated hepatitis B virus DNA is a source of HBsAg. Sci Transl Med. 2017;9(409):eaan0241 This paper reports early phase 2 data of RNAi in chronic hepatitis $B$ and subsequent work in chimpanzees to investigate the differential response in different cohorts of patients.

31.• Yuen MF, Schiefke I, Yoon JH, et al. RNA interference therapy with ARC-520 results in prolonged HBsAg response in patients with chronic hepatitis B infection. Hepatology. 2019. https:/doi. org/10.1002/hep. 31008 This phase 2 trial of RNAi in chronic hepatitis B demonstrates a persistence of qHBsAg to day 85 after last dose, however reductions are only modest.

32. Dusheiko G. Current and future directions of management of hepatitis B: steps toward a cure. Futur Virol. 2018;13(3):189-209.

33. Agarwal K, Gane E, Cheng W, et al. HBcrAg, HBV-RNA declines in a phase 2a study evaluating the multi-dose activity of ARB-1467 in $\mathrm{HBeAg}$-positive and negative virally suppressed subjects with hepatitis B. Hepatology. 2017;66(1).
34. Esau C, Limphong P, Tachikawa K, et al. LUNAR (TM)-HBV, a UNA oligomer combination for the treatment of chronic hepatitis $\mathrm{B}$ virus infection. Hepatology. 2016;63(1):912A-3A.

35. Han K, Cremer J, Elston R, Oliver S, Baptiste-Brown S, Chen S, et al. A randomized, double-blind, placebo-controlled, first-time-inhuman study to assess the safety, tolerability, and pharmacokinetics of single and multiple ascending doses of GSK3389404 in healthy subjects. Clinic Pharm Drug Dev. 2019;8(6):790-801.

36.• Loomba R, Morgan E, Watts L, et al. Novel antisense inhibition of diacylglycerol O-acyltransferase 2 for treatment of non-alcoholic fatty liver disease: a multicentre, double-blind, randomised, placebo-controlled phase 2 trial. Lancet Gastroenterol. 2020. https://doi.org/10.1016/S2468-1253(20)30186-2 This recent study has reported promising phase 2 data for RNAi in nonalcoholic fatty liver disease, utilizing a platform which is currently in clinical trials for chronic hepatitis $B$.

37. Gane EJ, Locarnini S, Lim TH, et al. Dose response with the RNA interference (RNAi) therapy JNJ-3989 combined with nucleos (t) ide analogue (NA) treatment in expanded cohorts of patients (PTS) with chronic hepatitis B (CHB). Hepatology. 2019;70(6):434A$5 \mathrm{~A}$.

38. Yuen MF, Locarnini S, Given B, et al. First clinical experience with RNA interference-based triple combination therapy in chronic hepatitis B: JNJ-3989, JNJ-6379 and a Nucleos (t) ide analogue. Hepatology. 2019;70(6):1489A.

39. Carey I, Gersch J, Wang B, Moigboi C, Kuhns M, Cloherty G, et al. Pre-genomic HBV RNA and HBcrAg predict outcomes in $\mathrm{HBeAg}$ negative chronic hepatitis B patients suppressed on Nucleos $(\mathrm{t})$ ide analogue therapy. Hepatology. 2019;72:42-57. https://doi.org/10. 1002/hep.31026.

40. Gane E, Locarnini S, Lim TH, et al. Short-term treatment with RNA interference therapy, JNJ-3989, results in sustained hepatitis B surface antigen suppression in patients with chronic hepatitis B receiving nucleos ( $\mathrm{t}$ ) ide analogue treatment. J Hepatol. 2020;73(S20).

Publisher's Note Springer Nature remains neutral with regard to jurisdictional claims in published maps and institutional affiliations. 